\title{
IGG4-RELATED DISEASE - DIAGNOSE FROM A PSEUDOTUMOR RETROPERITONIAL: A CASE REPORT
}

Roberta Fernandes Braz ${ }^{1, \star}$, João Vitor Santos Calzavara² ${ }^{2}$ Carolline Santos Aguiar Monção³

1.Centro Universitário FIPMoc, Montes Claros (MG), Brazil. 2.Universidade Estadual de Montes Claros, Montes Claros (MG), Brazil. 3.Universidade Federal de Minas Gerais, Belo Horizonte (MG), Brazil.

*Corresponding author: robertabraz@gmail.com

\section{BACKGROUND}

Related disease to immunoglobulin G4 (DR-lgG4) is an uncommon fibroinflammatory immune condition, which can affect multiple organs and share pathological, serological and clinical characteristics with several other diseases, that makes the diagnosis of this pathology a major clinical challenge. The organs most commonly affected are the pancreas, biliary tract, large salivary glands, lacrimal glands, retroperitoneum and lymph nodes. The pathogenesis of DR-lgG4 is still poorly understood, with autoimmunity and infectious processes being considered potential immunological triggers.

\section{CASE REPORT}

A male patient, 65 years old, smoker, alcoholic, complaining of left lower back pain, irradiation to the left iliac fossa, polyuria, feeling of incomplete bladder emptying, fever $\left(38^{\circ} \mathrm{C}\right)$, nausea and lack of appetite four days earlier. Physical examination, no fever, eupneic, flat abdomen, painful on palpation in the left iliac fossa and positive Giordano sign. Routine urine tests and urine culture showed no signs of infection. Complete blood count with normal leucocyte count and elevated C-reactive protein. Computed tomography of the abdomen showing a small mass with soft tissue density in the region of the internal iliac chain on the left, to be clarified, in close contact with distal ureter, conditioning a small hydroureteronephrosis. Magnetic resonance imaging of the abdomen showed an expansive/infiltrative lesion in the left pelvis, of neoplastic aspect, involving internal iliac vessels and distal ureter. Diagnosis of invasive retroperitoneal tumor, where it is not possible to exclude malignancy. The patient underwent laparoscopy: a large hardened mass in topography of the obturator and internal iliac fossa, making laparoscopic resection impossible. Conversion to open surgery, with complete resection of the lesion, internal iliac artery and vein and distal ureter. The biopsy result was unspecific, fusocellular proliferation permeated by adipose tissue and fibroblasts, and inflammatory cells. The hypothesis of DR-IgG4 was raised, serum IgG4 immunoglobulin measurement presented a result above the reference value, corroborating the hypothesis. Immunohistochemical study of the surgical piece was performed for diagnostic confirmation, revealing an increase in the number of positive lgG4 plasmocytes, a predominance of T lymphocytes (CD3+) and negativity of the other mesenchymal and epithelial markers. Undergoing corticosteroid therapy (prednisone $40 \mathrm{mg} / \mathrm{day}$ ) and referred for outpatient follow-up.

\section{CONCLUSION}

It is necessary to alert health professionals about the diagnosis of an unusual presentation of IgG4-related disease, such as a retroperitoneal pseudotumor. It is an important differential diagnosis, since it has a completely different prognosis and treatment than the usual malignant neoplasms that cause most retroperitoneal tumors. 